\title{
QUASI-SOCLE IDEALS IN LOCAL RINGS WITH GORENSTEIN TANGENT CONES
}

\author{
SHIRO GOTO, SATORU KIMURA, NAOYUKI MATSUOKA, AND TRAN THI PHUONG
}

\begin{abstract}
Quasi-socle ideals, that is the ideals $I$ of the form $I=Q: \mathfrak{m}^{q}$ in a Noetherian local ring $(A, \mathfrak{m})$ with the Gorenstein tangent cone $\mathrm{G}(\mathfrak{m})=\bigoplus_{n \geq 0} \mathfrak{m}^{n} / \mathfrak{m}^{n+1}$ are explored, where $q \geq 1$ is an integer and $Q$ is a parameter ideal of $A$ generated by monomials of a system $x_{1}, x_{2}, \cdots, x_{d}$ of elements in $A$ such that $\left(x_{1}, x_{2}, \cdots, x_{d}\right)$ is a reduction of $\mathfrak{m}$. The questions of when $I$ is integral over $Q$ and of when the graded rings $\mathrm{G}(I)=\bigoplus_{n \geq 0} I^{n} / I^{n+1}$ and $\mathrm{F}(I)=\bigoplus_{n \geq 0} I^{n} / \mathfrak{m} I^{n}$ are Cohen-Macaulay are answered. Criteria for $\mathrm{G}(I)$ and $\mathcal{R}(I)=\bigoplus_{n \geq 0} I^{n}$ to be Gorenstein rings are given.
\end{abstract}

\section{INTRODUCTION}

This paper aims at a study of quasi-socle ideals in a local ring with the Gorenstein tangent cone. Our purpose is to answer Question 1.1 below, of when the graded rings associated to the ideals are Cohen-Macaulay and/or Gorenstein rings, estimating their reduction numbers with respect to minimal reductions.

Let $A$ be a Noetherian local ring with the maximal ideal $\mathfrak{m}$ and $d=\operatorname{dim} A>0$. Let $Q=\left(x_{1}, x_{2}, \cdots, x_{d}\right)$ be a parameter ideal in $A$ and let $q \geq 1$ be an integer. We put $I=Q: \mathfrak{m}^{q}$ and refer to those ideals as quasi-socle ideals in $A$. Then one can ask the following questions, which are the main subject of the researches [GMT, GKM] and the present research as well.

\section{Question 1.1.}

(1) Find the conditions under which $I \subseteq \bar{Q}$, where $\bar{Q}$ stands for the integral closure of $Q$.

(2) When $I \subseteq \bar{Q}$, estimate or describe the reduction number

$$
\mathrm{r}_{Q}(I)=\min \left\{0 \leq n \in \mathbb{Z} \mid I^{n+1}=Q I^{n}\right\}
$$

Key words and phrases: Quasi-socle ideal, regular local ring, Cohen-Macaulay ring, Gorenstein ring, associated graded ring, Rees algebra, Fiber cone, integral closure.

2000 Mathematics Subject Classification: 13H10, 13A30, 13B22, $13 \mathrm{H} 15$. 
of $I$ with respect to $Q$ in terms of some invariants of $Q$ or $A$.

(3) Clarify what kind of ring-theoretic properties of the graded rings associated to the ideal $I$

enjoy.

$$
\mathcal{R}(I)=\bigoplus_{n \geq 0} I^{n}, \mathrm{G}(I)=\bigoplus_{n \geq 0} I^{n} / I^{n+1}, \text { and } \mathrm{F}(I)=\bigoplus_{n \geq 0} I^{n} / \mathfrak{m} I^{n}
$$

In this paper we shall focus our attention on a certain special kind of quasi-socle ideals. We now assume that the tangent cone, that is the associated graded ring

$$
\mathrm{G}(\mathfrak{m})=\bigoplus_{n \geq 0} \mathfrak{m}^{n} / \mathfrak{m}^{n+1}
$$

of $\mathfrak{m}$, is a Gorenstein ring and that the maximal ideal $\mathfrak{m}$ contains a system $x_{1}, x_{2}, \cdots, x_{d}$ of elements such that the ideal $\left(x_{1}, x_{2}, \cdots, x_{d}\right)$ is a reduction of $\mathfrak{m}$ (the latter condition is always satisfied if the field $A / \mathfrak{m}$ is infinite; see $[\mathrm{NR}]$ for the existence of reductions of $\mathfrak{m}$ generated by $d$ elements). Let $a_{1}, a_{2}, \cdots, a_{d}$, and $q$ be positive integers and we put

$$
Q=\left(x_{1}^{a_{1}}, x_{2}^{a_{2}}, \cdots, x_{d}^{a_{d}}\right) \text { and } I=Q: \mathfrak{m}^{q} .
$$

Let $\bar{A}=A / Q, \overline{\mathfrak{m}}=\mathfrak{m} / Q$, and $\bar{I}=I / Q$. Let $\rho=\max \left\{n \in \mathbb{Z} \mid \overline{\mathfrak{m}}^{n} \neq(0)\right\}$, that is index of nilpotency of the ideal $\overline{\mathfrak{m}}$, and put

$$
\ell=\rho+1-q
$$

We then have the following, which are the answers to Question 1.1 in our specific setting.

Theorem 1.2. The following three conditions are equivalent to each other.

(1) $I \subseteq \bar{Q}$.

(2) $\mathfrak{m}^{q} I=\mathfrak{m}^{q} Q$.

(3) $\ell \geq a_{i}$ for all $1 \leq i \leq d$.

When this is the case, the following assertions hold true.

(i) $\mathrm{r}_{Q}(I)=\left\lceil\frac{q}{\ell}\right\rceil:=\min \left\{n \in \mathbb{Z} \mid \frac{q}{\ell} \leq n\right\}$.

(ii) The graded rings $\mathrm{G}(I)$ and $\mathrm{F}(I)$ are Cohen-Macaulay.

Theorem 1.3. Suppose that $\ell \geq a_{i}$ for all $1 \leq i \leq d$. Then we have the following.

(i) $\mathrm{G}(I)$ is a Gorenstein ring if and only if $\ell \mid q$.

(ii) $\mathcal{R}(I)$ is a Gorenstein ring if and only if $q=(d-2) \ell$. 
Our setting naturally contains the case where $A$ is a regular local ring with $x_{1}, x_{2}, \cdots, x_{d}$ a regular system of parameters, the case where $A$ is an abstract hypersurface with the infinite residue class field, and the case where $A=R_{M}$ is the localization of the homogeneous Gorenstein ring $R=k\left[R_{1}\right]$ over an infinite field $k=R_{0}$ at the irrelevant maximal ideal $M=R_{+}$. In Section 3 we will explore a few examples, including these three cases, in order to see how Theorems 1.2 and 1.3 work for the analysis of concrete examples. The proofs of Theorems 1.2 and 1.3 themselves shall be given in Section 2. However, before entering details, let us here explain the reason why we are interested in quasi-socle ideals.

The study of socle ideals dates back to a research of L. Burch [B], where she explored certain socle ideals of finite projective dimension and gave a characterization of regular local rings (cf. GH, Theorem 1.1]). More recently, A. Corso and C. Polini [CP1, CP2] studied, with interaction to the linkage theory of ideals, the socle ideals $I=Q: \mathfrak{m}$ in a Cohen-Macaulay local ring $(A, \mathfrak{m})$ and showed that $I^{2}=Q I$, once $A$ is not a regular local ring. Consequently, the rings $\mathrm{G}(I)$ and $\mathrm{F}(I)$ are Cohen-Macaulay and so is the ring $\mathcal{R}(I)$ if $\operatorname{dim} A \geq 2$. The first author and H. Sakurai [GSa1, GSa2, GSa3] explored also the case where the base ring is not necessarily Cohen-Macaulay but Buchsbaum, and showed that the equality $I^{2}=Q I$ (here $I=Q: \mathfrak{m}$ ) holds true for numerous parameter ideals $Q$ in a given Buchsbaum local ring $(A, \mathfrak{m})$, whence $\mathrm{G}(I)$ is a Buchsbaum ring, provided that $\operatorname{dim} A \geq 2$ or that $\operatorname{dim} A=1$ but the multiplicity $\mathrm{e}_{\mathfrak{m}}^{0}(A)$ of $A$ with respect to $\mathfrak{m}$ is not less than 2 .

A more important thing is, however, the following. If $J$ is an equimultiple CohenMacaulay ideal of reduction number one in a Cohen-Macaulay local ring, the associated graded ring $\mathrm{G}(J)=\bigoplus_{n \geq 0} J^{n} / J^{n+1}$ of $J$ is a Cohen-Macaulay ring and, so is the Rees algebra $\mathcal{R}(J)=\bigoplus_{n \geq 0} J^{n}$ of $J$, provided $\mathrm{ht}_{A} J \geq 2$. One knows the number and degrees of defining equations of $\mathcal{R}(J)$ also, which makes the process of desingularization of $\operatorname{Spec} A$ along the subscheme $\mathrm{V}(J)$ fairly explicit to understand. This observation motivated the ingenious research of C. Polini and B. Ulrich [PU], where they posed, among many important results, the following conjecture. 
Conjecture $1.4([\mathrm{PU}])$. Let $(A, \mathfrak{m})$ be a Cohen-Macaulay local ring with $\operatorname{dim} A \geq 2$. Assume that $\operatorname{dim} A \geq 3$ when $A$ is regular. Let $q \geq 2$ be an integer and let $Q$ be a parameter ideal in $A$ such that $Q \subseteq \mathfrak{m}^{q}$. Then

$$
Q: \mathfrak{m}^{q} \subseteq \mathfrak{m}^{q}
$$

This conjecture was settled by H.-J. Wang [Wan, whose theorem says:

Theorem 1.5 ([Wan] $)$. Let $(A, \mathfrak{m})$ be a Cohen-Macaulay local ring with $d=\operatorname{dim} A \geq 2$. Let $q \geq 1$ be an integer and $Q$ a parameter ideal in $A$. Assume that $Q \subseteq \mathfrak{m}^{q}$ and put $I=Q: \mathfrak{m}^{q}$. Then

$$
I \subseteq \mathfrak{m}^{q}, \quad \mathfrak{m}^{q} I=\mathfrak{m}^{q} Q, \quad \text { and } \quad I^{2}=Q I,
$$

provided that $A$ is not regular if $d \geq 2$ and that $q \geq 2$ if $d \geq 3$.

The recent research [GMT] of the first and the third authors jointly with R. Takahashi reports a different approach to the Polini-Ulrich Conjecture 1.4 and has proven the following.

Theorem 1.6 (GMT]). Let $(A, \mathfrak{m})$ be a Gorenstein local ring with $d=\operatorname{dim} A>0$ and $\mathrm{e}_{\mathfrak{m}}^{0}(A) \geq 3$, where $\mathrm{e}_{\mathfrak{m}}^{0}(A)$ denotes the multiplicity of $A$ with respect to $\mathfrak{m}$. Let $Q$ be a parameter ideal in $A$ and put $I=Q: \mathfrak{m}^{2}$. Then $\mathfrak{m}^{2} I=\mathfrak{m}^{2} Q, I^{3}=Q I^{2}$, and $\mathrm{G}(I)=\bigoplus_{n \geq 0} I^{n} / I^{n+1}$ is a Cohen-Macaulay ring, so that $\mathcal{R}(I)=\bigoplus_{n \geq 0} I^{n}$ is also a Cohen-Macaulay ring, provided $d \geq 3$.

The researches [Wan and [GMT] are performed independently and their methods of proof are totally different from each other's. The technique of [GMT] can not go beyond the restrictions that $A$ is a Gorenstein ring, $q=2$, and $\mathrm{e}_{\mathfrak{m}}^{0}(A) \geq 3$. However, despite these restrictions, the result GMT, Theorem 1.1] holds true even in the case where $\operatorname{dim} A=1$, while Wang's result says nothing about the case where $\operatorname{dim} A=$ 1. As is suggested in [GMT, the one-dimensional case is substantially different from higher-dimensional cases and more complicated to control. This observation has led the first three authors to the research [GKM], where they have explored quasi-socle ideals in Gorenstein numerical semigroup rings over fields as the first step towards further investigations of the Polini-Ulrich Conjecture 1.4 of arbitrary dimension. The present 
research is, more or less, a continuation of [Wan, GMT, GKM], and our Theorems 1.2 and 1.3 might have some significance towards the second step, providing some insight with Question 1.1, that are not presented by [Wan, GMT, GKM].

\section{Proof of Theorems 1.2 And 1.3}

The purpose of this section is to prove Theorems 1.2 and 1.3. First of all, let us restate our setting, which we shall maintain throughout this paper.

Let $A$ be a Noetherian local ring with the maximal ideal $\mathfrak{m}$ and $d=\operatorname{dim} A>0$. We assume that the associated graded ring

$$
\mathrm{G}(\mathfrak{m})=\bigoplus_{n \geq 0} \mathfrak{m}^{n} / \mathfrak{m}^{n+1}
$$

of $\mathfrak{m}$ is Gorenstein and that the maximal ideal $\mathfrak{m}$ contains a system $x_{1}, x_{2}, \cdots, x_{d}$ of elements which generates a reduction of $\mathfrak{m}$. Then, since $G(\mathfrak{m})$ is a Gorenstein ring, the base local ring $A$ is also Gorenstein and the initial forms $\left\{X_{i}\right\}_{1 \leq i \leq d}$ of $\left\{x_{i}\right\}_{1 \leq i \leq d}$ with respect to $\mathfrak{m}$ constitute a regular sequence in $G(\mathfrak{m})$, so that we get a canonical isomorphism

$$
\mathrm{G}\left(\mathfrak{m} /\left(x_{1}, x_{2}, \cdots, x_{d}\right)\right) \cong \mathrm{G}(\mathfrak{m}) /\left(X_{1}, X_{2}, \cdots, X_{d}\right)
$$

of graded $A$-algebras $([\mathrm{VV}])$. Let $a_{1}, a_{2}, \cdots, a_{d}$, and $q$ be positive integers and we put

$$
Q=\left(x_{1}^{a_{1}}, x_{2}^{a_{2}}, \cdots, x_{d}^{a_{d}}\right) \text { and } I=Q: \mathfrak{m}^{q} .
$$

Let $\bar{A}=A / Q, \overline{\mathfrak{m}}=\mathfrak{m} / Q$, and $\bar{I}=I / Q$. Then

$$
\mathrm{G}(\overline{\mathfrak{m}}) \cong \mathrm{G}(\mathfrak{m}) /\left(X_{1}^{a_{1}}, X_{2}^{a_{2}}, \cdots, X_{d}^{a_{d}}\right),
$$

whence $\mathrm{G}(\overline{\mathfrak{m}})$ is a Gorenstein ring. Let $\rho=\max \left\{n \in \mathbb{Z} \mid \overline{\mathfrak{m}}^{n} \neq(0)\right\}$, that is index of nilpotency of the ideal $\overline{\mathfrak{m}}$, and we have

$$
\rho=\mathrm{a}(\mathrm{G}(\overline{\mathfrak{m}}))=\mathrm{a}(\mathrm{G}(\mathfrak{m}))+\sum_{i=1}^{d} a_{i},
$$

where a $(*)$ denotes the $a$-invariant of the corresponding graded ring ([GW, (3.1.4)]).

Let

$$
\ell=\rho+1-q
$$

By [Wat] (see [O, Theorem 1.6] also) we then have the following. 
Proposition 2.1. (0): $\overline{\mathfrak{m}}^{i}=\overline{\mathfrak{m}}^{\rho+1-i}$ for all $i \in \mathbb{Z}$. In particular $\bar{I}=(0): \overline{\mathfrak{m}}^{q}=\overline{\mathfrak{m}}^{\ell}$ whence $I=Q+\mathfrak{m}^{\ell}$.

The key for our proof of Theorem 1.2 is the following.

Lemma 2.2. Suppose that $\ell \geq a_{i}$ for all $1 \leq i \leq d$. Then

$$
Q \cap \mathfrak{m}^{n \ell+m} \subseteq \mathfrak{m}^{m} Q I^{n-1}
$$

for all $m \geq 0$ and $n \geq 1$.

Proof. We have

$$
Q \cap \mathfrak{m}^{n \ell+m}=\sum_{i=1}^{d} x_{i}^{a_{i}} \mathfrak{m}^{n \ell+m-a_{i}}
$$

since $x_{1}, x_{2}, \cdots, x_{d}$ is a super regular sequence with respect to $\mathfrak{m}$ (that is, their initial forms $X_{1}, X_{2}, \cdots, X_{d}$ constitute a regular sequence in $\left.\mathrm{G}(\mathfrak{m})\right)$. Because

$$
n \ell+m-a_{i}=(n-1) \ell+m+\left(\ell-a_{i}\right) \geq(n-1) \ell+m
$$

for each $1 \leq i \leq d$, we get

$$
\mathfrak{m}^{n \ell+m-a_{i}} \subseteq \mathfrak{m}^{(n-1) \ell+m}=\mathfrak{m}^{m} \cdot\left(\mathfrak{m}^{\ell}\right)^{n-1} .
$$

Therefore, since $\mathfrak{m}^{\ell} \subseteq I$ by Proposition 2.1, we have

$$
\begin{aligned}
Q \cap \mathfrak{m}^{n \ell+m} & =\sum_{i=1}^{d} x_{i}^{a_{i}} \mathfrak{m}^{n \ell+m-a_{i}} \\
& \subseteq \sum_{i=1}^{d} x_{i}^{a_{i}} \mathfrak{m}^{m}\left(\mathfrak{m}^{\ell}\right)^{n-1} \\
& \subseteq \mathfrak{m}^{m} Q I^{n-1}
\end{aligned}
$$

as is claimed.

Let us now prove Theorem 1.2.

Proof of Theorem 1.2. (2) $\Rightarrow$ (1) This is well-known. See [NR, Section 7, Theorem 2].

$(3) \Rightarrow(2)$ By Proposition 2.1 we get $\mathfrak{m}^{q} I=\mathfrak{m}^{q} Q+\mathfrak{m}^{q+\ell}$, whence $\mathfrak{m}^{q+\ell} \subseteq Q$, so that $\mathfrak{m}^{q+\ell}=Q \cap \mathfrak{m}^{q+\ell} \subseteq \mathfrak{m}^{q} Q$ by Lemma 2.2 , because $\ell \geq a_{i}$ for all $1 \leq i \leq d$. Thus $\mathfrak{m}^{q} I=\mathfrak{m}^{q} Q$. 
$(1) \Rightarrow(3)$ Let $1 \leq i \leq d$ be an integer. Then $x_{i}^{\ell} \in \mathfrak{m}^{\ell} \subseteq I \subseteq \bar{Q}$. We look at the integral equation

$$
\left(x_{i}^{\ell}\right)^{n}+c_{1}\left(x_{i}^{\ell}\right)^{n-1}+\cdots+c_{n}=0
$$

with $n \gg 0$ and $c_{j} \in Q^{j}$. Then $x_{i}^{n \ell} \in \sum_{j=1}^{n} Q^{j} x_{i}^{(n-j) \ell}$ and so, thanks to the monomial property of the sequence $x_{1}, x_{2}, \cdots, x_{d}$ (cf. [E, Exercise 17.13, c.]; recall that the sequence $x_{1}, x_{2}, \cdots, x_{d}$ is $A$-regular), we have

$$
x_{i}^{n \ell} \in Q^{j} x_{i}^{(n-j) \ell}
$$

for some $1 \leq j \leq n$. Let $L=\left\{\left(\alpha_{1}, \alpha_{2}, \cdots, \alpha_{d}\right) \in \mathbb{Z}^{d} \mid \alpha_{k} \geq 0\right.$ for all $\left.1 \leq k \leq d\right\}$ and $\Lambda_{j}=\left\{\alpha \in L \mid \sum_{k=1}^{d} \alpha_{k}=j\right\}$. Then since

$$
Q^{j}=\left(\prod_{k=1}^{d} x_{k}^{a_{k} \alpha_{k}} \mid \alpha \in \Lambda_{j}\right),
$$

thanks to the monomial property of $x_{1}, x_{2}, \cdots, x_{d}$ again, we get

$$
x_{i}^{n \ell} \in\left[\prod_{k=1}^{d} x_{k}{ }^{a_{k} \alpha_{k}}\right] \cdot x_{i}^{(n-j) \ell} A
$$

for some $\alpha \in \Lambda_{j}$. Hence

$$
(n \ell) \mathbf{e}_{i}=\sum_{k=1}^{d}\left(a_{k} \alpha_{k}\right) \mathbf{e}_{k}+[(n-j) \ell] \mathbf{e}_{i}+\beta
$$

with $\beta \in L$, where $\left\{\mathbf{e}_{i}\right\}_{1 \leq i \leq d}$ denotes the standard basis of $\mathbb{Z}^{d}$. Consequently $\alpha_{k}=\beta_{k}=$ 0 if $k \neq i$ and so $\alpha_{i}=j$. Hence $0=a_{i} j-j \ell+\beta_{i}$, so that we have $\ell \geq a_{i}$ as is claimed.

Let us now consider assertions (i) and (ii). Let $n \geq 1$ be an integer. Then $I^{n}=$ $Q I^{n-1}+\mathfrak{m}^{n \ell}$ since $I=Q+\mathfrak{m}^{\ell}$ (Proposition 2.1), so that

$$
Q \cap I^{n}=Q I^{n-1}+\left[Q \cap \mathfrak{m}^{n \ell}\right] \subseteq Q I^{n-1}
$$

because $Q \cap \mathfrak{m}^{n \ell} \subseteq Q I^{n-1}$ by Lemma 2.2. Therefore $Q \cap I^{n}=Q I^{n-1}$ for all $n \geq 1$, whence $\mathrm{G}(I)$ is a Cohen-Macaulay ring ([VV, Corollary 2.7]).

We will show that $\mathrm{r}_{Q}(I)=\left\lceil\frac{q}{\ell}\right\rceil$. Notice that

$$
\mathrm{r}_{Q}(I)=\min \left\{n \geq 0 \mid I^{n+1} \subseteq Q\right\}
$$


because $I^{n+1}=Q I^{n}$ if and only if $I^{n+1} \subseteq Q$. Firstly, suppose that $I^{n+1} \subseteq Q$. We then have $\overline{\mathfrak{m}}^{(n+1) \ell}=(0)$ (recall that $\bar{I}=\overline{\mathfrak{m}}^{\ell}$ ), whence $(n+1) \ell \geq \rho+1$. Therefore

$$
n+1 \geq \frac{\rho+1}{\ell}=\frac{q+\ell}{\ell}=\frac{q}{\ell}+1
$$

because $\ell=\rho+1-q$, so that we have $n \geq \frac{q}{\ell}$.

If $n \geq \frac{q}{\ell}$, then $(n+1) \ell \geq\left(\frac{q}{\ell}+1\right) \ell=q+\ell=\rho+1$ and so $\bar{I}^{n+1}=\overline{\mathfrak{m}}^{(n+1) \ell}=(0)$, whence $I^{n+1} \subseteq Q$. Thus $\mathrm{r}_{Q}(I)=\left\lceil\frac{q}{\ell}\right\rceil$.

Because $\mathrm{G}(I)$ is a Cohen-Macaulay ring, to see the fiber cone $\mathrm{F}(I)$ is Cohen-Macaulay it suffices to show that

$$
Q \cap \mathfrak{m} I^{n}=\mathfrak{m} Q I^{n-1}
$$

for all $n \geq 1$ (see, e.g., CGPU, CZ]). By Lemma 2.2 we have

$$
\begin{aligned}
Q \cap \mathfrak{m} I^{n} & =Q \cap\left[\mathfrak{m} Q I^{n-1}+\mathfrak{m}^{n \ell+1}\right] \\
& =\mathfrak{m} Q I^{n-1}+\left[Q \cap \mathfrak{m}^{n \ell+1}\right] \\
& \subseteq \mathfrak{m} Q I^{n-1},
\end{aligned}
$$

whence $Q \cap \mathfrak{m} I^{n}=\mathfrak{m} Q I^{n-1}$.

Assume that $\ell \geq a_{i}$ for all $1 \leq i \leq d$ and let $Y_{i}^{\prime}$ 's be the initial forms of $x_{i}^{a_{i}}$ 's with respect to $I$. Then $Y_{1}, Y_{2}, \cdots, Y_{d}$ is a homogeneous system of parameters of $\mathrm{G}(I)$, since $Q$ is a reduction of $I$ (Theorem 1.2). It therefore constitutes a regular sequence in $\mathrm{G}(I)$, because $\mathrm{G}(I)$ is a Cohen-Macaulay ring by Theorem 1.2 (ii), so that we have a canonical isomorphism

$$
\mathrm{G}(\bar{I}) \cong \mathrm{G}(I) /\left(Y_{1}, Y_{2}, \cdots, Y_{d}\right)
$$

of graded $A$-algebras $([\mathrm{VV}])$. Hence a $(\mathrm{G}(\bar{I}))=\mathrm{a}(\mathrm{G}(I))+d$. Let $r$ be the index of nilpotency of $\bar{I}$, that is $r=\mathrm{a}(\mathrm{G}(\bar{I}))$. Then since $r=\mathrm{r}_{Q}(I)$ (recall that $x_{1}{ }^{a_{1}}, x_{2}{ }^{a_{2}}, \cdots, x_{d}{ }^{a_{d}}$ is a super regular sequence with respect to $I)$ and $\mathrm{a}(\mathrm{G}(I))=\mathrm{a}(\mathrm{G}(\bar{I}))-d([\mathrm{GW},(3.1 .6)])$, by Theorem 1.2 (i) we have the following.

Lemma 2.3. Suppose that $\ell \geq a_{i}$ for all $1 \leq i \leq d$. Then $\mathrm{a}(\mathrm{G}(I))=\left\lceil\frac{q}{\ell}\right\rceil-d$.

Corollary 2.4. Assume that $\ell \geq a_{i}$ for all $1 \leq i \leq d$. Then $\mathcal{R}(I)$ is a Cohen-Macaulay ring if and only if $\left\lceil\frac{q}{\ell}\right\rceil<d$. When this is the case, $d \geq 2$. 
Proof. Since $\mathrm{G}(I)$ is a Cohen-Macaulay ring by Theorem 1.2 (ii), $\mathcal{R}(I)$ is a CohenMacaulay ring if and only if $a(\mathrm{G}(I))<0$ ([TI]). By Lemma 2.3 the latter condition is equivalent to saying that $\left\lceil\frac{q}{\ell}\right\rceil<d$ (cf. [GSh, Remark (3.10)]). When this is case, $d \geq 2$ because $0<\left\lceil\frac{q}{\ell}\right\rceil$.

We are now in a position to prove Theorem 1.3 .

Proof of Theorem 1.3. (i) Notice that $\mathrm{G}(I)$ is a Gorenstein ring if and only if so is the graded ring

$$
\mathrm{G}(\bar{I})=\mathrm{G}(I) /\left(Y_{1}, Y_{2}, \cdots, Y_{d}\right)
$$

where $Y_{i}$ 's stand for the initial forms of $x_{i}^{a_{i}}$ 's with respect to $I$. Let $r$ be the index of nilpotency of $\bar{I}$. Then $r=\mathrm{r}_{Q}(I)=\left\lceil\frac{q}{\ell}\right\rceil$, and $\mathrm{G}(\bar{I})$ is a Gorenstein ring if and only if the equality

$$
\text { (0) }: \bar{I}^{i}=\bar{I}^{r+1-i}
$$

holds true for all $i \in \mathbb{Z}([\mathrm{O}$, Theorem 1.6]). Hence if $\mathrm{G}(I)$ is a Gorenstein ring, we have (0) $: \bar{I}=\bar{I}^{r}=\overline{\mathfrak{m}}^{r \ell}$. On the other hand, since $\bar{I}=\overline{\mathfrak{m}}^{\ell}$ and $q=\rho+1-\ell$, by Proposition 2.1 we get

$$
\text { (0) }: \bar{I}=(0): \overline{\mathfrak{m}}^{\ell}=\overline{\mathfrak{m}}^{q} .
$$

Therefore $q=r \ell$, since $\overline{\mathfrak{m}}^{r \ell}=\overline{\mathfrak{m}}^{q} \neq(0)$. Thus $\ell \mid q$ and $r=\frac{q}{\ell}$.

Conversely, suppose that $\ell \mid q$. Hence $r=\frac{q}{\ell}$ by Theorem 1.2 (i). Let $i \in \mathbb{Z}$. Then since $\bar{I}=\overline{\mathfrak{m}}^{\ell}$, we get $\bar{I}^{r+1-i}=\overline{\mathfrak{m}}^{(r+1-i) \ell}$, while

$$
(0): \bar{I}^{i}=(0): \overline{\mathfrak{m}}^{i \ell}=\overline{\mathfrak{m}}^{\rho+1-i \ell}
$$

by Proposition 2.1. We then have (0) $: \bar{I}^{i}=\bar{I}^{r+1-i}$ for all $i \in \mathbb{Z}$, since

$$
(r+1-i) \ell=q+\ell-i \ell=\rho+1-i \ell
$$

Thus $\mathrm{G}(\bar{I})$ is a Gorenstein ring, whence so is $\mathrm{G}(I)$.

(ii) The Rees algebra $\mathcal{R}(I)$ of $I$ is a Gorenstein ring if and only if $\mathrm{G}(I)$ is a Gorenstein ring and $a(\mathrm{G}(I))=-2$, provided $d \geq 2([\underline{\mathbb{I}}$, Corollary (3.7)]). Suppose that $\mathcal{R}(I)$ is a Gorenstein ring. Then $d \geq 2$ by Corollary 2.4. Since $a(\mathrm{G}(I))=\mathrm{r}_{Q}(I)-d=-2$, by assertion (i) and Theorem 1.2 (i) we have $\frac{q}{\ell}=\mathrm{r}_{Q}(I)=d-2$, whence $q=(d-2) \ell$. Conversely, suppose that $q=(d-2) \ell$. Then $d \geq 3$ since $q \geq 1$. By assertion (i) and 
Theorem 1.2 (i) $\mathrm{G}(I)$ is a Gorenstein ring with $\mathrm{r}_{Q}(I)=\frac{q}{\ell}=d-2$, whence $a(\mathrm{G}(I))=$ $(d-2)-d=-2$, so that $\mathcal{R}(I)$ is a Gorenstein ring.

Example 2.5. Suppose that $\rho \geq 5$ is an odd integer, say $\rho=2 \tau+1$ with $\tau \geq 2$. Let $q=\rho-1$. Then $\ell=\rho+1-q=2$. Hence, choosing $a_{i} \leq 2$ for all $1 \leq i \leq d$, we have $I=Q+\mathfrak{m}^{2} \subseteq \bar{Q}$ with $\mathrm{r}_{Q}(I)=\tau$ by Theorem 1.2. Since $\ell \mid q$, by Theorem 1.3 (i) the ring $\mathrm{G}(I)$ is Gorenstein, despite $Q \nsubseteq \mathfrak{m}^{q}$ (compare the condition with those in Theorem 1.5). The ring $\mathcal{R}(I)$ is by Theorem 1.3 (ii) a Gorenstein ring, if $d=\tau+2$.

\section{EXAMPLES AND APPLiCATIONS}

In this section we shall discuss some applications of Theorems 1.2 and 1.3, Let us begin with the case where $A$ is a regular local ring.

3.1. The case where $A$ is a regular local ring. Let $A$ be a regular local ring with $x_{1}, x_{2}, \cdots, x_{d}$ a regular system of parameters. Similarly as in the previous sections, let

$$
Q=\left(x_{1}^{a_{1}}, x_{2}^{a_{2}}, \cdots, x_{d}^{a_{d}}\right) \text { and } I=Q: \mathfrak{m}^{q}
$$

with positive integers $a_{1}, a_{2}, \cdots, a_{d}$, and $q$. Then $\mathrm{G}(\mathfrak{m})=k\left[X_{1}, X_{2}, \cdots, X_{d}\right]$ is the polynomial ring, where $k=A / \mathfrak{m}$ and $X_{i}$ 's are the initial forms of $x_{i}$ 's, so that we have

$$
\rho=\sum_{i=1}^{d} a_{i}-d \text { and } \ell=\sum_{i=1}^{d}\left(a_{i}-1\right)+1-q,
$$

since $\mathrm{a}(\mathrm{G}(\mathfrak{m}))=-d$. Notice that the condition that

$$
\ell \geq \max \left\{a_{i} \mid 1 \leq i \leq d\right\}
$$

is equivalent to saying that

$$
\sum_{j \neq i} a_{j} \geq q+d-1
$$

for all $1 \leq i \leq d$, because $\ell-a_{i}=\sum_{j \neq i} a_{j}-(q+d-1)$. When this is the case, $d \geq 2$.

We readily get, thanks to Theorem 1.2 , the following.

Example 3.1. The following assertions hold true.

(1) Let $d=2$. Then $I \subseteq \bar{Q}$ if and only if $\min \left\{a_{1}, a_{2}\right\} \geq q+1$.

(2) Let $d=3$. Then $I \subseteq \bar{Q}$ if and only if $\min \left\{a_{i}+a_{j} \mid 1 \leq i<j \leq 3\right\} \geq q+2$. 
(3) Choose integers $a$ and $q$ so that $2 \leq a \leq d$ and $(d-1)(a-1)<q \leq d(a-1)$. Let $a_{i}=a$ for all $1 \leq i \leq d$. Then $I \subsetneq A$ but $I \nsubseteq \bar{Q}$. For example, let $d=3, a=2$, and $q=3$. Then

$$
\left(x_{1}^{2}, x_{2}^{2}, x_{3}^{2}\right): \mathfrak{m}^{3}=\mathfrak{m} \nsubseteq \overline{\left(x_{1}^{2}, x_{2}^{2}, x_{3}^{2}\right)} .
$$

Thanks to Theorem 1.3, we are able to produce quasi-socle ideals $I=Q: \mathfrak{m}^{q}$ whose Rees algebras are Gorenstein.

Example 3.2. The following assertions hold true.

(1) Let $d=2$ and assume that $I \subseteq \bar{Q}$. Then $\mathrm{G}(I)$ is not a Gorenstein ring.

(2) Suppose that $d \geq 3$ and let $n \geq d-1$ be an integer. Let $a_{1}=d-1, a_{i}=n$ for all $2 \leq i \leq d$, and $q=(d-2) n$. Then $\mathcal{R}(I)$ is a Gorenstein ring.

(3) Suppose that $d=5$ and let $a_{i}=4$ for all $1 \leq i \leq 5$. Let $q=8$. Then $I \subseteq \bar{Q}$ and $\mathrm{G}(I)$ is a Gorenstein ring with $\mathrm{r}_{Q}(I)=1$, but $\mathcal{R}(I)$ is not a Gorenstein ring.

Proof. (1) Suppose that $\mathrm{G}(I)$ is a Gorenstein ring and let $q=r \ell$ with $r=\mathrm{r}_{Q}(I)$. Then $a_{1}+a_{2}-1=\rho+1=q+\ell=\ell(r+1)$, which implies, because $\ell \geq a_{i}$ for $i=1,2$ by Theorem 1.2, that $r=0$. This is impossible.

(2) Since $\rho=n d-n-1$, we get $\ell=n$. Hence $q=(d-2) \ell$, so that by Theorem 1.3 (ii) $\mathcal{R}(I)$ is a Gorenstein ring.

(3) We have $\rho=15$ and $\ell=q=8$. Hence $I \subseteq \bar{Q}$ with $\mathrm{r}_{Q}(I)=1$ by Theorem 1.2. By Theorem 1.3 $\mathrm{G}(I)$ is a Gorenstein ring, but the ring $\mathcal{R}(I)$ is not, because $q \neq(d-2) \ell$.

Remark 3.3. When $A$ is not a regular local ring, the associated graded $\operatorname{ring} \mathrm{G}(I)$ of $I$ can be Gorenstein, even though $d=2$. See Example 3.9.

Since the base ring $A$ is regular, the Cohen-Macaulayness in Rees algebras $\mathcal{R}(I)$ follows from that of associated graded rings $\mathrm{G}(I)([\mathrm{L}])$. Let us note a brief proof in our context.

Proposition 3.4. Suppose that $\ell \geq a_{i}$ for all $1 \leq i \leq d$. Then the Rees algebra $\mathcal{R}(I)$ is a Cohen-Macaulay ring. 
Proof. By Corollary 2.4 we have only to show $\left\lceil\frac{q}{\ell}\right\rceil<d$. Let $a_{k}=\max \left\{a_{i} \mid 1 \leq i \leq d\right\}$. Then because $\ell \geq a_{k}$, we have

$$
\frac{q}{\ell}+1=\frac{\rho+1}{\ell} \leq \frac{\sum_{j=1}^{d}\left(a_{j}-1\right)+1}{a_{k}}=\sum_{j \neq k} \frac{a_{j}-1}{a_{k}}+1<d,
$$

whence $\left\lceil\frac{q}{\ell}\right\rceil<d$ as is wanted.

Let $L=\left\{\left(\alpha_{1}, \alpha_{2}, \cdots, \alpha_{d}\right) \in \mathbb{Z}^{d} \mid \alpha_{i} \geq 0\right.$ for all1 $\left.\leq i \leq d\right\}$. For each $\alpha=$ $\left(\alpha_{1}, \alpha_{2}, \cdots, \alpha_{d}\right) \in L$ we put $x^{\alpha}=\prod_{i=1}^{d} x_{i}^{\alpha_{i}}$. Let $\mathfrak{a}$ be an ideal in $A$. Then we say that $\mathfrak{a}$ is a monomial ideal, if $\mathfrak{a}$ is generated by monomials in $\left\{x_{i}\right\}_{1 \leq i \leq d}$, that is $\mathfrak{a}=\left(x^{\alpha} \mid \alpha \in \Lambda\right)$ for some $\Lambda \subseteq L$. Monomial ideals behave very well as if they were monomial ideals in the polynomial ring $k\left[x_{1}, x_{2}, \cdots, x_{d}\right]$ over a field $k$ (see [HS] for details). For instance, the integral closure $\bar{Q}$ of our monomial ideal $Q$ is also a monomial ideal and we have the following.

Proposition $3.5([\mathrm{HS}])$. Let $\Delta=\left\{\alpha \in L \mid \sum_{i=1}^{d} \frac{\alpha_{i}}{a_{i}} \geq 1\right\}$. Then $\bar{Q}=\left(x^{\alpha} \mid \alpha \in \Delta\right)$.

Corollary 3.6. Suppose that $d \geq 2$ and let $n \geq 2$ be an integer. We put $\mathfrak{q}=$ $\left(x_{1}^{n-1}, x_{2}^{n}, \cdots, x_{d}^{n}\right)$. Then $\overline{\mathfrak{q}}=\mathfrak{q}+\mathfrak{m}^{n}=\left(x_{1}^{n-1}\right)+\mathfrak{m}^{n}$ and all the powers $\overline{\mathfrak{q}}^{m}(m \geq 1)$ are integrally closed.

Proof. Let $J=\mathfrak{q}+\mathfrak{m}^{n}$ and $\mathfrak{a}=\left(x_{1}^{n}, x_{2}^{n}, \cdots, x_{d}^{n}\right)$. Then $\mathfrak{a} \subseteq \mathfrak{q}$ and $\mathfrak{m}^{n} \subseteq \overline{\mathfrak{a}}$, so that $J \subseteq \overline{\mathfrak{q}}$. Let $m \geq 1$ be an integer and put $K=\left(x_{1}^{m(n-1)}, x_{2}^{m n}, \cdots, x_{d}^{m n}\right)$. We will show that $\bar{K} \subseteq J^{m}$. Let $\alpha \in L$ and assume that $\frac{\alpha_{1}}{m(n-1)}+\sum_{i=2}^{d} \frac{\alpha_{i}}{m n} \geq 1$. We want to show that $x^{\alpha} \in J^{m}$. We may assume that $\alpha_{1}<m(n-1)$. Let $\alpha_{1}=(n-1) i+j$ with $i, j \in \mathbb{Z}$ such that $0 \leq j<(n-1)$. Then $0 \leq i<m$. Since $\frac{\alpha_{1}}{m(n-1)}+\sum_{i=2}^{d} \frac{\alpha_{i}}{m n} \geq 1$, we get

$$
n \alpha_{1}+(n-1) \cdot \sum_{i=2}^{d} \alpha_{i} \geq m n(n-1)
$$

so that

$$
(n-1) \cdot \sum_{i=2}^{d} \alpha_{i} \geq m n(n-1)-n \alpha_{1}=n[(n-1)(m-i)-j]
$$

whence

$$
\sum_{i=2}^{d} \alpha_{i} \geq n(m-i)-\frac{n j}{n-1}
$$


Because $\frac{n j}{n-1}=j+\frac{j}{n-1}$ and $0 \leq j<n-1$, we have $\frac{n j}{n-1}=j+\frac{j}{n-1}<j+1$ and so

$$
\sum_{i=2}^{d} \alpha_{i} \geq n(m-i)-j
$$

Thus

$$
x^{\alpha}=x_{1}^{(n-1) i} \cdot x_{1}^{j} x_{2}^{\alpha_{2}} \cdots x_{d}^{\alpha_{d}} \in x_{1}^{(n-1) i} \mathfrak{m}^{n(m-i)} \subseteq J^{m},
$$

whence $\bar{K} \subseteq J^{m}$ by Proposition 3.5 .

Because $J^{m} \subseteq \overline{\mathfrak{q}}^{m}$ and $\mathfrak{q}^{m} \subseteq \bar{K}$, we have $J^{m} \subseteq \overline{\mathfrak{q}}^{m} \subseteq \overline{\mathfrak{q}}^{m} \subseteq \bar{K}$, whence $J^{m}=\overline{\mathfrak{q}}^{m}=$ $\overline{\mathfrak{q}^{m}}=K$. Letting $m=1$, we get $J=\overline{\mathfrak{q}}$. This completes the proof of Corollary 3.6.

Thanks to Corollary 3.6, we get the following characterization for quasi-socle ideals $I=Q: \mathfrak{m}^{q}$ to be integrally closed.

Proposition 3.7. Suppose that $d \geq 2$ and $a_{i} \geq 2$ for all $1 \leq i \leq d$. Then the following two conditions are equivalent to each other.

(1) $I=\bar{Q}$.

(2) Either (a) $a_{i}=\ell$ for all $1 \leq i \leq d$, or (b) there exists $1 \leq j \leq d$ such that $a_{i}=\ell$ if $i \neq j$ and $a_{j}=\ell-1$.

When this is the case, $\overline{I^{n}}=I^{n}$ for all $n \geq 1$, whence $\mathcal{R}(I)$ is a Cohen-Macaulay normal domain.

Proof. (1) $\Rightarrow(2)$ Since $I=\bar{Q}$, we get $q \leq \rho$ and $I=Q+\mathfrak{m}^{\ell}$ (Proposition 2.1). Notice that

$$
Q \subseteq I=Q: \mathfrak{m}^{q} \subsetneq\left(Q: \mathfrak{m}^{q}\right): \mathfrak{m}=Q: \mathfrak{m}^{q+1}
$$

because $I \subsetneq A$. Hence $Q: \mathfrak{m}^{q+1} \nsubseteq \bar{Q}$. Consequently $\ell-1=\rho+1-(q+1)<a_{i}$ for some $1 \leq i \leq d$ by Theorem 1.2 , so that, thanks to Theorem 1.2 again, we have

$$
\ell=a_{i} \geq a_{j}
$$

for all $1 \leq j \leq d$. Let $\Delta=\left\{1 \leq j \leq d \mid a_{j}<\ell\right\}$. We then have the following.

Claim. (1) $a_{j}=\ell-1$, if $j \in \Delta$.

(2) $\sharp \Delta \leq 1$. 
Proof. Let $j \in \Delta$. Then $a_{j}<\ell=a_{i}$ whence $j \neq i$ and $\ell \geq 3$. Let $\alpha=\left(a_{j}-1\right) \mathbf{e}_{j}+\left(a_{i}-\right.$ $\left.a_{j}\right) \mathbf{e}_{i}$. Then $\alpha \in L$ but, thanks to the monomial property of ideals, $x^{\alpha} \notin Q+\mathfrak{m}^{\ell}=I=\bar{Q}$, because $\sum_{k=1}^{d} \alpha_{k}=a_{i}-1=\ell-1$ and $x^{\alpha} \notin Q$. Consequently, $\sum_{k=1}^{d} \frac{\alpha_{k}}{a_{k}}<1$ by Proposition 3.5, so that $1<\frac{1}{a_{j}}+\frac{a_{j}}{a_{i}}$, because

$$
\frac{a_{j}-1}{a_{j}}+\frac{a_{i}-a_{j}}{a_{i}}<1
$$

Let $n=a_{i}-a_{j}$. Then $a_{j}\left(a_{i}-a_{j}\right)<a_{i}$ as $1<\frac{1}{a_{j}}+\frac{a_{j}}{a_{i}}$, whence $a_{j} n<a_{i}=a_{j}+n$ so that $0 \leq\left(a_{j}-1\right)(n-1)<1$. Hence $n=1$ (recall that $a_{j} \geq 2$ ) and $a_{j}=a_{i}-1=\ell-1$.

Assume $\sharp \Delta \geq 2$ and choose $j, k \in \Delta$ so that $j \neq k$. We put $y=x_{j} x_{k}^{\ell-2}$. We then have $y^{\ell-1}=\left(x_{j}^{\ell-1}\right)\left(x_{k}^{\ell-1}\right)^{\ell-2}=\left(x_{j}^{a_{j}}\right)\left(x_{k}^{a_{k}}\right)^{\ell-2} \in Q^{\ell-1}$, because $a_{j}=a_{k}=\ell-1$ by assertion (1). Hence $y \in \bar{Q}=Q+\mathfrak{m}^{\ell}$, which is impossible because $y \notin Q$ (recall that $\ell \geq 3$ ) and $y \notin \mathfrak{m}^{\ell}$, thanks to the monomial property of ideals. Hence $\sharp \Delta \leq 1$.

If $\Delta=\emptyset$, we then have $\ell=a_{j}$ for all $1 \leq j \leq d$. If $\Delta \neq \emptyset$, letting $\Delta=\{j\}$, we get $a_{i}=\ell$ if $i \neq j$ and $a_{j}=\ell-1$. This proves the implication (1) $\Rightarrow(2)$.

$(2) \Rightarrow(1)$ Suppose condition (b) is satisfied. Then $I=Q+\mathfrak{m}^{\ell}=\left(x_{j}^{\ell-1}\right)+\mathfrak{m}^{\ell}=\bar{Q}$ by Proposition 2.1 and Corollary 3.6. Suppose condition (a) is satisfied. Then $I \subseteq \bar{Q}$ by Theorem 1.2 and $I=Q+\mathfrak{m}^{\ell}=\mathfrak{m}^{\ell}$ by Proposition 2.1, whence $I=\bar{Q}$. In each case all the powers of $I$ are integrally closed (see Corollary 3.6 for case (b)), whence the last assertion follows from Proposition 3.4.

Example 3.8. Suppose that $d \geq 3$ and let $n \geq d-1$ be an integer. We look at the ideal

$$
Q=\left(x_{1}^{d-1}, x_{2}^{n}, x_{3}^{n}, \cdots, x_{d}^{n}\right)
$$

and let $q=n(d-2)$. Then $\ell=n$, as $\rho=n d-(n+1)$, whence $I \subseteq \bar{Q}$ and $I=Q+\mathfrak{m}^{n}=$ $\left(x_{1}^{d-1}\right)+\mathfrak{m}^{n}$. The ring $\mathcal{R}(I)$ is by Theorem 1.3 (ii) a Gorenstein ring, since $q=(d-2) \ell$. If $n=d$, then $I=\left(x_{1}^{d-1}\right)+\mathfrak{m}^{d}$ and $\overline{I^{m}}=I^{m}$ for all $m \geq 1$ by Corollary [3.6, so that $\mathcal{R}(I)$ is a Gorenstein normal ring.

3.2. The case where $A=R_{M}$. Our setting naturally contains the case where $A=R_{M}$ is the localization of the homogeneous Gorenstein ring $R=k\left[R_{1}\right]$ over an infinite field $k=R_{0}$ at the irrelevant maximal ideal $M=R_{+}$. Let us note one example. 
Example 3.9. Let $S=k[X, Y, Z]$ be the polynomial ring over an infinite field $k$ and let $R=S / f S$, where $0 \neq f \in S$ is a form with degree $n \geq 2$. Then $R$ is a homogeneous Gorenstein ring with $\operatorname{dim} R=2$. Let $x_{1}, x_{2}$ be a linear system of parameters in $R$ and let $M=R_{+}$. We look at the local ring $A=R_{M}$. Let $a_{1}=2, a_{2}=n$, and $q=n$. Let $Q=\left(x_{1}^{2}, x_{2}^{n}\right) A$ and $I=Q: \mathfrak{m}^{q}$, where $\mathfrak{m}=M A$. Then

$$
\rho=\mathrm{a}(R)+\left(a_{1}+a_{2}\right)=2 n-1 .
$$

Hence $\ell=q=n$, so that $I \subseteq \bar{Q}, I=Q+\mathfrak{m}^{n}=\left(x_{1}^{2}\right)+\mathfrak{m}^{n}$, and $\mathrm{G}(I)$ is a Gorenstein ring with $\mathrm{r}_{Q}(I)=1$ (Theorems 1.2 and 1.3 ). We have $Q \nsubseteq \mathfrak{m}^{q}$, if $n \geq 3$.

3.3. The case where $A=k\left[\left[t^{a}, t^{b}\right]\right]$. Let $1<a<b$ be integers with $\operatorname{GCD}(a, b)=1$. We look at the ring $A=k\left[\left[t^{a}, t^{b}\right]\right] \subseteq k[[t]]$, where $k[[t]]$ denotes the formal powers series ring over a field $k$. We put $x=t^{a}$ and $y=t^{b}$. Then $A$ is a one-dimensional Gorenstein local ring and $\mathfrak{m}=(x, y)$. Because $A \cong k[[X, Y]] /\left(X^{b}-Y^{a}\right)$ where $k[[X, Y]]$ denotes the formal powers series ring over the field $k$, we get

$$
\mathrm{G}(\mathfrak{m}) \cong k[X, Y] /\left(Y^{a}\right)
$$

Let $n, q \geq 1$ be integers, and put $Q=\left(x^{n}\right)$ and $I=Q: \mathfrak{m}^{q}$. Then because $a(\mathrm{G}(\mathfrak{m}))=$ $a-2$, we have $\rho=a+n-2$ and $\ell=(a+n)-(q+1)$. Consequently $I \subseteq \bar{Q}$ if and only if $q<a$ (Theorem 1.2), whence the condition that $I \subseteq \bar{Q}$ is independent of the choice of the integer $n \geq 1$. When this is the case, by Theorems 1.2 and 1.3 we have the following.

Proposition 3.10. The following assertions hold true.

(1) $\mathrm{r}_{Q}(I)=\left\lceil\frac{q}{(a+n)-(q+1)}\right\rceil$.

(2) The graded rings $\mathrm{G}(I)$ and $\mathrm{F}(I)$ are Cohen-Macaulay rings.

(3) The ring $\mathrm{G}(I)$ is a Gorenstein ring if and only if $(a+n)-(q+1)$ divides $q$.

Hence, if $q=a-1$, we then have, for each integer $n \geq 1$ such that $n \mid q$, that $\mathrm{G}(I)$ is a Gorenstein ring.

\section{REFERENCES}

[B] L. Burch, On ideals of finite homological dimension in local rings, Proc. Camb. Phil. Soc., 64 (1968), 941-948. 
[CGPU] A. Corso, L. Ghezzhi, C. Polini, and B. Ulrich, Cohen-Macaulayness of special fiber rings, Comm. Algebra, 31 (2003), no. 8, 3713-3734.

[CZ] T. Cortadellas and S. Zarzuela, On the Cohen-Macaulay property of the fiber cone of ideals with reduction number at most one, Commutative algebra, algebraic geometry, and computational methods (Hanoi, 1996), 215-222, Springer, Singapore, 1999.

[CP1] A. Corso and C. Polini, Links of prime ideals and their Rees algebras, J. Algebra, 178 (1995), $224-238$.

[CP2] A. Corso and C. Polini, Reduction number of links of irreducible varieties, J. Pure Appl. Algebra, 121 (1997), 29-43.

[E] D. Eisenbud, Commutative Algebra with a View Toward Algebraic Geometry, Graduate Texts in Mathematics, 150. Springer-Verlag, New York, 1995

[GH] S. Goto and F. Hayasaka Finite homological dimension and primes associated to integrally closed ideals, Proc. Amer. Math. Soc., 130 (2002), 3159-3164.

[GKM] S. Goto, S. Kimura, and N. Matsuoka, Quasi-socle ideals in Gorenstein numerical semigroup rings, Preprint 2007.

[GMT] S. Goto, N. Matsuoka, and Ryo Takahashi, Quasi-socle ideals in a Gorenstein local ring, J. Pure App. Algebra (to appear).

[GSa1] S. Goto and H. Sakurai, The equality $I^{2}=Q I$ in Buchsbaum rings, Rend. Sem. Mat. Univ. Padova, 110 (2003), 25-56.

[GSa2] S. Goto and H. Sakurai, The reduction exponent of socle ideals associated to parameter ideals in a Buchsbaum local ring of multiplicity two, J. Math. Soc. Japan, 56 (2004), 1157-1168.

[GSa3] S. Goto and H. Sakurai, When does the equality $I^{2}=Q I$ hold true in Buchsbaum rings?, Commutative Algebra, 115-139, Lect. Notes Pure Appl. Math., 244, 2006.

[GSh] S. Goto and Y. Shimoda, On the Rees algebras of Cohen-Macaulay local rings, Commutative Algebra (Fairfax, Va., 1979), 201-231, Lecture Notes in Pure and Appl. M

[GW] S. Goto and K. Watanabe, On graded rings I, J. Math. Soc. Japan, 30(1978), 179-213. Proc. London Math. Soc., 29 (1974), 55-76.

[HS] R. Hübl, I. Swanson, Adjoints of ideals, arXiv:math.AC/0701071.

[I] S. Ikeda, On the Gorensteinness of Rees algebras over local rings, Nagoya Math. J., 102 (1986), $135-154$.

[L] J. Lipman, Cohen-Macaulayness in graded algebras, Math. Res. Lett., 1 (1994), no. 2, 149-157.

$[\mathrm{NR}]$ D. G. Northcott and D. Rees, Reductions of ideals in local rings, Proc. Camb. Phil. Soc., 50 (1954), 145-158.

[O] A. Ooishi, On the Gorenstein property of the associated graded ring and the Rees algebra of an ideal, J. Algebra, 115 (1993), 397-414.

[PU] C. Polini and B. Ulrich, Linkage and reduction numbers, Math. Ann., 310 (1998), 631-651.

[TI] N. V. Trung and S. Ikeda, When is the Rees algebra Cohen-Macaulay?, Comm. Algebra, 17 (1989), 2893-2922.

[VV] P. Valabrega and G. Valla, Form rings and regular sequences, Nagoya. Math. J., 72 (1978), 93-101.

[Wan] H.-J. Wang, Links of symbolic powers of prime ideals, Math. Z., 256 (2007), 749-756.

[Wat] J. Watanabe, The Dilworth number of Artin Gorenstein rings, Adv. in Math., 76 (1989), 194-199. 
Department of Mathematics, School of Science and Technology, Meiji University, 1-1-1 Higashimita, TAMA-KU, KaWASAKi 214-8571, JaPAN

E-mail address: goto@math.meiji.ac.jp

Department of Mathematics, School of Science and Technology, Meiji University, 1-1-1 Higashimita, TAMA-KU, KaWASAKi 214-8571, JaPAN

E-mail address: skimura@math.meiji.ac.jp

Department of Mathematics, School of Science and Technology, Meiji University, 1-1-1 Higashimita, TAMA-KU, KaWASAKi 214-8571, JaPAN

E-mail address: matsuoka@math.meiji.ac.jp

Department of Information Technology and Applied Mathematics, Ton Duc Thang University, 98 Ngo Tat To Street, Ward 19, Binh Thanh District, Ho Chi Minh City, VIETNAM

E-mail address: sugarphuong@gmail.com 